KOMPASS

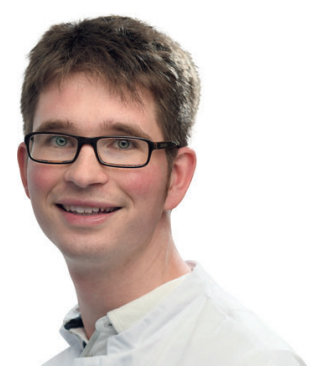

\section{Marcel Wiesweg}

Innere Klinik (Tumorforschung)

Universitätsklinikum Essen

Deutschland

\title{
Marcel Wiesweg
}

\section{Preisträger des «Takeda Oncology Forschungspreises» 2019}

\section{Forschungsschwerpunkte}

- NSCLC

- Analyse klinischer molekular charakterisierter Subgruppen

- Prädiktive Faktoren für Immuntherapie

\section{Homepage}

- https://tumorforschung.uk-essen.de/ueber-uns/unser-team/aerztliche-mitarbeiter/

Welches Problem bzw. welche Idee liegt Ihrer ausgezeichneten Forschungsarbeit «The Immunotarget registry: Immune checkpoint inhibitors for patients with advanced lung cancer and oncogenic driver alterations» zugrunde?

Die Fragestellung ist die Wirksamkeit der Immuntherapie (Anti-PD-1/PD-L1) beim nicht kleinzelligen Lungenkarzinom (non-small cell lung carcinoma, NSCLC) mit dominanten onkogenen Alterationen. Hierzu wurde ein internationales Netzwerk von 24 Zentren in 10 Ländern aufgebaut. Maßgebliche Initiatoren waren Julien Mazières aus Toulouse, Oliver Gautschi aus Luzern und Alexander Drilon aus New York. Die Verläufe von Patienten unter Immuntherapie mit metastasiertem Lungenkarzinom und Alterationen von KRAS, EGFR, ALK, ROS1, BRAF, MET, RET oder HER2 wurden retrospektiv erfasst.

Worin bestanden die größten Herausforderungen bei der Konzeption und Entwicklung Ihres Forschungsansatzes?

Zum einen handelt es sich (mit Ausnahme von KRAS und EGFR) um doch recht geringe Patientenzahlen, so dass selbst mit einem großen internationalen Netzwerk nur Subgruppen mit Patientenzahlen im unteren bis mittleren zweistelligen Bereich erreicht werden können. Zum anderen liegt es in der Natur einer retrospektiven Studie, dass die Datenqualität variiert und so Informationen wie z.B. zum Raucherstatus oder zum PD-L1-Status des Tumors nur unvollständig vorhanden sind. Insgesamt ergeben sich trotz des sehr großen Gesamtkollektivs von 551 Patienten in der Analyse Probleme, die die Aussagekraft einschränken.

Was hat Sie an den Ergebnissen Ihrer Untersuchung am meisten überrascht? Es handelte sich um die erste wirklich umfassende Arbeit zu diesem Thema, die in mancherlei Hinsicht das Bild geprägt hat und durch andere Arbeiten ergänzt wird. Sehr deutlich wurde z.B. die extrem geringen Ansprechraten von Tumoren mit ALK- oder RET-Translokation. Zudem wurden einige Details herausgearbeitet, wie etwa die Assoziation von Ansprechen auf Immuntherapie und vorherigem Nikotinkonsum bei BRAF-mutierten Lungenkarzinomen, die für die individuelle Beratung von Interesse sein können.

\section{KARGER}

Fax +497614520714 information@karger.com www.karger.com
() 2019 S. Karger GmbH, Freiburg
Dr. Marcel Wiesweg

Innere Klinik (Tumorforschung)

Universitätsklinikum Essen

Hufelandstr. 55, 45147 Essen, Deutschland

marcel.wiesweg@uk-essen.de 
Wo zeigt sich in Ihrem Ansatz die Relevanz für die klinische Praxis besonders?

Man muss sicher einschränkend sagen, dass sich zwischenzeitlich die Standardtherapie geändert hat und die große Mehrheit der Patienten in der Erstlinie eine Chemo-Immunkombination erhält Gleichzeitig begründen die Daten erneut den Ausschluss von Patienten mit EGFR- und ALK-Alteration von dieser Erstlinientherapie. Relevanz erhalten die Daten insbesondere bei der Wichtung der Immuntherapie bei vortherapierten, IO-naiven Patienten.

Was ist aus Ihrer Erfahrung wichtig für die Schnittstelle zwischen Forschung und klinischer Medizin?

Für Patienten mit metastasiertem NSCLC ist zunächst entscheidend, dass der Tumor auch wirklich umfassend molekularpathologisch untersucht wird. Mit dieser Voraussetzung können alle Fortschritte der Präzisionsonkologie erst beim Patienten ankommen. Hervorzuheben ist hier das nationale Netzwerk Genomische Medizin (nNGM), das neben dem Angebot zur molekularen Untersuchung auch darauf abzielt, klinische Forschung und klinische Studien bestmöglich zu integrieren.
Der 1. Preis ist mit 30000 EUR dotiert. Verraten Sie uns, wofür Sie das Preisgeld verwenden möchten?

Da wir hier nur Teil eines internationalen Netzwerkes sind, wird ein Anteil des Preisgeldes sicherlich für infrastrukturelle Maßnahmen und weitere Projekte international und an unserem Zentrum eingesetzt werden.

Herr Dr. Wiesweg, wir bedanken uns herzlich für das Interview!

\section{ANGABEN ZUM PREIS}

Unter dem Motto «Unser Antrieb: Innovationen fördern» ruft Takeda Oncology Kliniken und klinische Einrichtungen dazu auf, herausragende Projekte aus dem Bereich «NSCLC-Oncogene Addicted» für den mit insgesamt 60000 EUR dotierten TakedaForschungspreis einzureichen. Auch 2019 wurden exzellente Arbeiten aus der präklinischen und klinischen Forschung von einem unabhängigen Gremium prämiert.

Der Forschungspreis wurde 2018 erstmals ausgeschrieben und soll durch die Auszeichnung wegweisender Projekte Innovationen fördern. Er wird im Rahmen eines Takeda-Symposiums bei der Jahrestagung der Deutschen Gesellschaft für Hämatologie und Medizinische Onkologie (DGHO) verliehen.

www.takeda-onkologie.de/takeda-oncology-forschungspreisnsclc-2019 